\title{
A rare case of an unexpected difficult airway management in a diffuse idiopathic skeletal hyperostosis patient and post-operative airway evaluation with 3D printing technique
}

\author{
Xiaoqing Zhang, Jun Wang, Yajie Liu, Zhengqian Li, Bin Han \\ Department of Anesthesiology, Peking University Third Hospital, Beijing, China \\ Correspondence to: Bin Han. Department of Anesthesiology, Peking University Third Hospital, 49 North Garden Rd., Haidian District, Beijing \\ 100191, China. Email: hanbinputh@163.com.
}

\begin{abstract}
Diffuse idiopathic skeletal hyperostosis (DISH), asymptomatic in most cases, is a degenerative condition that commonly leads to anterior cervical osteophytes in most elderly patients. Clinically significant airway compression is rare. However, in some cases, the seemingly insignificant osteophytes could become a threat to airway management during intubation. Here we present a case of an 82-year-old man diagnosed as DISH and scheduled for cervical surgery. Radiographic imaging did not indicate the airway compromise. Preoperative airway assessment indicated modified Mallampati scoring of class III. However, an unexpected large bulge protruding from posterior pharyngeal wall blocked video laryngoscopy assisted intubation. Intubation with direct laryngoscope, laryngeal mask airway (LMAD) and Shikani optical stylet were failed. Only flexible fiberoptic nasal intubation was performed successfully. After surgery, an anatomically matched and patient-specific 3D printed model was made to help more direct and comprehensive estimation of the size and the location of the osteophyte. The relationship of the airway compromise and osteophytes was revealed in DISH patients for the first time with the aid of 3D printed model. The eccentric growth of the cervical osteophytes occupied large portion of the laryngopharynx space and prohibited the direct placement of the tracheal tube. This case is a rare presentation of the management of a DISH patient with unexpected difficult airway. We propose for the first time that different intubation strategies should be considered for potential difficulty airway in DISH patients according to the size and location of the osteophytes. For potential difficult airway management of DISH patients, $3 \mathrm{D}$ printing technique is a promising way of preoperative airway assessment.
\end{abstract}

Keywords: 3D printing; diffuse idiopathic skeletal hyperostosis (DISH); fiberoptic bronchoscopy; difficult airway; cervical vertebra

Submitted Aug 20, 2020. Accepted for publication Nov 01, 2020.

doi: $10.21037 / \mathrm{atm}-20-5992$

View this article at: http://dx.doi.org/10.21037/atm-20-5992

\section{Introduction}

Diffuse idiopathic skeletal hyperostosis (DISH) is a degenerative condition that commonly leads to anterior cervical osteophytes. A small percentage of patients develop dysphagia, cough, dyspnea, and dysphonia. The reported prevalence of DISH in literature is quite variable ranging from $2.9 \%$ to $28 \%(1,2)$. As most elderly patients with significant degenerative changes involving the disk and the osteophytes tend to be asymptomatic in most cases, some authors consider the abnormalities as radiographic findings rather than a disease. Non-operative management is the mainstay of the treatment. In cases of failed non-operative modalities, surgical resection of the osteophytes has been suggested to be the last resort. Clinically significant airway compression is rare. However, in some cases, the seemingly insignificant osteophytes could become a threat to airway management during intubation. We present the following case in accordance with the CARE reporting checklist 

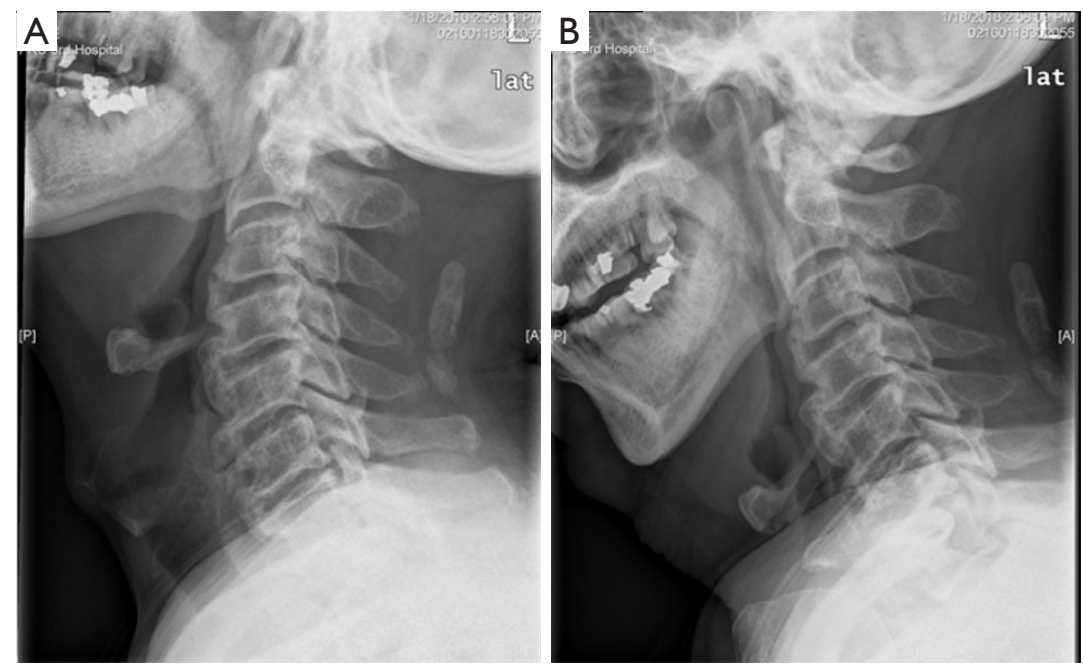

Figure 1 Cervical X-ray of the patient with diffuse idiopathic skeletal hyperostosis disease in extension (A) and flexion (B) position.

(available at http://dx.doi.org/10.21037/atm-20-5992).

\section{Case presentation}

An 82-year-old man weighing $73 \mathrm{~kg}$, who suffered from progressive dysphagia for 3 years and worsened during the last 1 year, was diagnosed with "DISH, cervical disc herniation, anterior longitudinal ligament ossification, and cervical spinal canal stenosis". He was scheduled for elective anterior cervical discectomy and fusion operation. His past surgical history included the posterior lumbar interbody fusion for lumbar canal stenosis and the left nephrectomy for renal mass 12 and 4 years ago, respectively. Examination and investigations were otherwise unremarkable.

Previous anesthetic records did not reveal difficult airway, and the patient has a clear medical history of otorhinolaryngology for the past few years. The patient showed no symptoms of dyspnea, stridor and OSAS. Modified Mallampati test was graded as class III. Other airway evaluation parameters were determined to be in the normal range. X-radiographs of cervical vertebra in extension and flexion position revealed the presence of new bone formation at cervical spine level C3-7 (Figure 1). No significant narrowing of trachea lumen was noticed in radiological findings.

The patient's $\mathrm{SpO}_{2}$ was $96 \%$ breathing room air. Following sufficient facemask preoxygenation, the patient was sedated intravenously. Considering the adequacy of facemask ventilation, the muscle relaxant was given. At the initial attempt with video laryngoscopy, the unexpected presence of a large bulge protruding from the posterior pharyngeal wall was revealed. The epiglottis and the vocal cords could not be identified. The bulge was rigid and non-pulsatile. It partially obstructed the airway and prevented endotracheal intubation. Attempts to reposition the video laryngoscopy failed and other efforts including direct laryngoscope with thin blade and the use of optical stylet or laryngeal mask airway (LMAD) were to no avail. Non-emergent airway was immediately recognized and a reinforced endotracheal tube with sealing cuff $(7.5 \mathrm{~mm}$ I.D.) lubricated with benoxinate gel was successfully intubated from nostril under fiberoptic bronchoscope (FOB) guidance. Video laryngoscopy inspection revealed the tracheal tube passing through the narrowing gap between the left pharyngeal wall and the large osteophytes (Figure 2).

The surgery was performed with dissection of $\mathrm{C} 4-7$ osteophytes and fusion of C5-6 and C6-7, leaving C3-4 osteophytes unremoved as surgeons believed that it was not the culprit of the dysphagia. The tracheal tube was extubated 40 hours postoperatively in fear of tracheal edema. The patient was discharged from intensive care unit and hospital uneventfully after 2 and 5 days, respectively. Considering the rarity of the difficult airway condition related with DISH disease, we retrospectively made a $3 \mathrm{D}$ printed model to help revealing the airway structure.

All procedures performed in studies involving human participant was in accordance with the ethical standards of the institutional and national research committee and with the Helsinki Declaration (as revised in 2013). Written informed consent was obtained from the patient. 


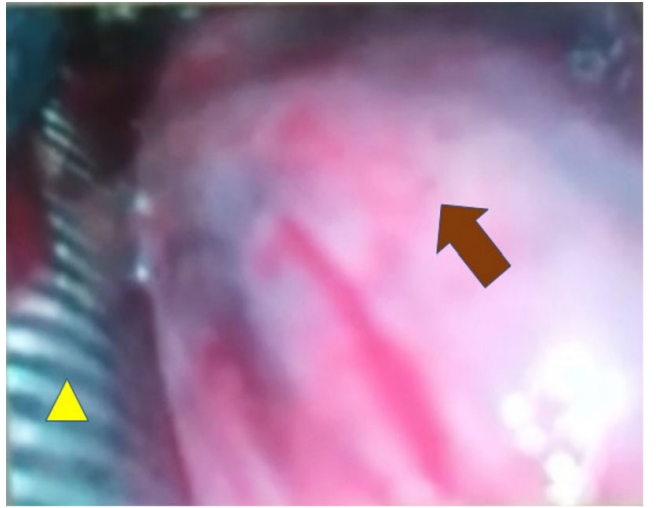

Figure 2 Video laryngoscopy inspection revealed the tracheal tube passing through the narrowing gap between the left pharyngeal wall and the large osteophytes. Triangle, tracheal tube; arrow, large protruding osteophytes.

\section{Discussion}

DISH is a systemic skeletal disease characterized by ligamentous ossification of the anterolateral spine and the extraspinal ligaments. There is no classification system for the severity of DISH. The extent of the disease process and the degree of ossification can vary significantly, and there is no direct correlation between the size of the osteophytes and the severity of dysphagia (3). For patients with DISH disease requiring surgical operation, the number of reported incidences of difficult tracheal intubation was scarce compared with the number of operated cases.

The unanticipated difficulties in tracheal intubation is frequently encountered even with comprehensive airway evaluation. It is currently recognized that bedside tests to predict difficult intubation with direct laryngoscopy have limited sensitivity and specificity, especially in the general population of patients with unknown pathology of the airway structure (4). Preoperative reviewing of the radiological images of DISH patients is important. The severity of the airway compromise is dependent upon the vertebrae levels involved and the amount and orientation of the bone deposited. The protruding osteophytes anteriorly could impinge upon the posterior wall of pharynx, and cause narrowing at the corresponding laryngopharynx and trachea (5). Lateral radiological imaging could help identifying "bony" structure and could be used as an indicator for preoperative airway assessment. However, the eccentric growth of the osteophytes, the overall trachea structure, and their relationship, cannot be fully reflected by the lateral view of $\mathrm{X}$-ray.
It is a pity that we did not predict this case as difficult airway based on preoperative 2D radiological imagines, past surgical history, and bedside airway evaluation. 3D radiological image reconstruction was made after surgery using computerized tomography imaging. It could help understanding the location, the severity of the osteophytes and the airway compromise in overall perspective, $3 \mathrm{D}$ printing has been well integrated in all fields of medical practice and literature reports. Printed models have been widely applied in analyzing complex cases, simulating procedures and implanting grafts $(6,7)$. To our knowledge, this is the first time that the relationship of the osteophytes and the airway structure of the DISH patient was clearly revealed with $3 \mathrm{D}$ printing technique.

$3 \mathrm{D}$ printing model is a cost-effective graspable entity. This technique enables the production of an anatomically matched and patient-specific model with high fidelity and complexity. By rotating, disassembling and assembling the model, we could accurately analyze the size and the range of the osteophyte bulge and its close relationship with the trachea. While the drawback of the current $3 \mathrm{D}$ printing technique is that there lacks suitable material to restore the authentic texture of the complex tissues (ligaments and joints). It cannot simulate the cervical range of motion during extension and flexion of cervical spine, either. This requires the evaluation with 2D images of $\mathrm{X}$-ray. Progress in this field will, without doubt, may provide more valuable information for preoperative airway evaluation, and help anesthesiologists choose the best intubation method and suitable kits in advance. In the current case, the eccentric growth of the cervical osteophytes (corresponding to C3-4 prevertebral osteophytes) occupy large portion of the middle and right laryngopharynx space, and caused difficult intubation (Figure 3).

For DISH patients, we proposed for the first time that different intubation strategies and tools should be carefully considered according to the location and severity of the osteophytes. For C2-3 and C3-4 osteophytes, the osseous protrusion could cause reduced oropharyngeal space, impaired epiglottic motility, the distortion of the laryngeal cartilages and the pharyngeal structure, and may interfere with laryngeal function, as was demonstrated by the current case (8). Therefore, for prominent osteophytes at proximal level of $\mathrm{C} 2-4$, it is recommended to intubate with FOB assistance (9). The osteophytes at the proximal level may diminish the laryngeal space, which is a potential threat to intubation. LMA may not be a good choice as the osteophytes at the supraglottic level inhibit its right 

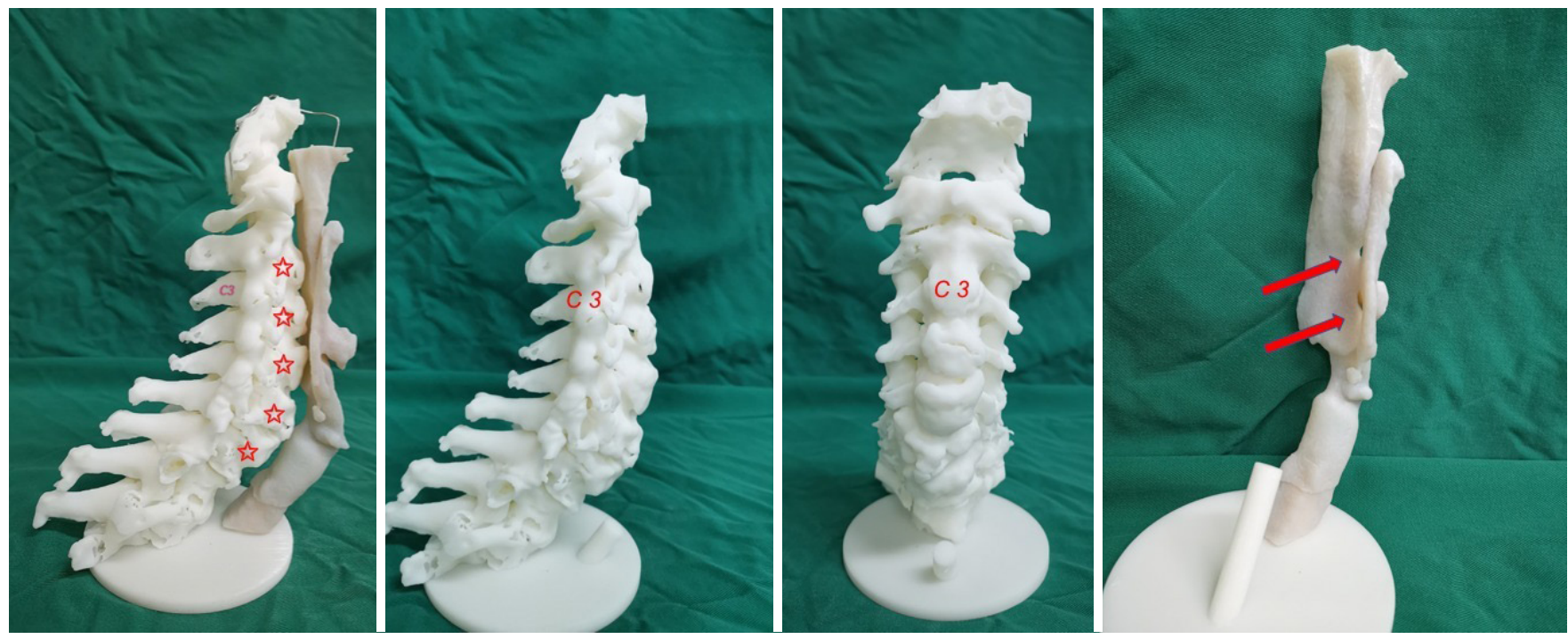

Figure 3 3D printing model revealing the relationship between the giant cervical osteophytes and airway compromise. C3, the 3rd cervical vertebra. Pentagram, large osteophytes could be clearly revealed in C3-7 that caused difficult intubation; arrow, indented airway caused by protruding osteophytes from $\mathrm{C} 3$ to $\mathrm{C} 7$.

positioning. It is reported that osteophytes in C4-5 resulted in laryngeal spasms and breathing cessation when bending the patient's head. Therefore, the "head-in-line" position should be maintained to prevent laryngeal spasms attack (10). Careful preoperative bedside assessment of the patient's head bending backward and radiological images are important for estimating the severity of the difficult airway. Awake intubation under FOB guidance should be adopted for osteophytes located above epiglottis. Osteophytes at the distal level may cause obstruction below the vocal cords. Preoperative airway evaluation should be emphasized on the degree of tracheal stenosis and selection of suitable tube size. LMA is recommended as a safe guarantee for sufficient ventilation. In this situation, FOB-assisted intubation could be performed with the aid of intubating laryngeal mask airway (ILMA).

To our knowledge, this is the first DISH model with $3 \mathrm{D}$ printing technique that could help recognizing the interrelationship between the bony structure and trachea. It is a pity that we could only retrospectively make an airway 3D model and assess the airway compromise. For future DISH patients with suspected difficult airway, especially those with large, eccentric, and protruding osteophytes that cause clinical symptoms such as severe dysphagia or syncope, careful preoperative evaluation with $3 \mathrm{D}$ printing method should be emphasized. By providing graspable entities that are patient-specific and with high fidelity, 3D printing technique could help anesthesiologists to fully appreciate the anatomical structure of the trachea and to avoid life-threatening conditions during difficult intubation.

\section{Acknowledgments}

Funding: This work was supported by "The capital health research and development of special”, grant number 20162-4092.

\section{Footnote}

Reporting Checklist: The authors have completed the CARE reporting checklist. Available at http://dx.doi.org/10.21037/ atm-20-5992

Conflicts of Interest: All authors have completed the ICMJE uniform disclosure form (available at http://dx.doi. org/10.21037/atm-20-5992). The authors have no conflicts of interest to declare.

Ethical Statement: The authors are accountable for all aspects of the work in ensuring that questions related to the accuracy or integrity of any part of the work are appropriately investigated and resolved. The ethical committee of Peking University Third Hospital waived the requirement for approval to conduct this single 
retrospective case study with access to medical records. All procedures performed in studies involving human participant was in accordance with the ethical standards of the institutional and national research committee and with the Helsinki Declaration (as revised in 2013). Written informed consent was obtained from the patient.

Open Access Statement: This is an Open Access article distributed in accordance with the Creative Commons Attribution-NonCommercial-NoDerivs 4.0 International License (CC BY-NC-ND 4.0), which permits the noncommercial replication and distribution of the article with the strict proviso that no changes or edits are made and the original work is properly cited (including links to both the formal publication through the relevant DOI and the license). See: https://creativecommons.org/licenses/by-nc-nd/4.0/.

\section{References}

1. Weinfeld RM, Olson PN, Maki DD, et al. The prevalence of diffuse idiopathic skeletal hyperostosis (DISH) in two large American Midwest metropolitan hospital populations. Skeletal Radiol 1997;26:222-5.

2. Kim SK, Choi BR, Kim CG, et al. The prevalence of diffuse idiopathic skeletal hyperostosis in Korea. J Rheumatol 2004;31:2032-5.

3. Seidler TO, Pèrez Alvarez JC, Wonneberger K, et al.

Cite this article as: Zhang X, Wang J, Liu Y, Li Z, Han B. A rare case of an unexpected difficult airway management in a diffuse idiopathic skeletal hyperostosis patient and postoperative airway evaluation with 3D printing technique. Ann Transl Med 2021;9(1):75. doi: 10.21037/atm-20-5992
Dysphagia caused by ventral osteophytes of the cervical spine: clinical and radiographic findings. Eur Arch Otorhinolaryngol 2009;266:285-91.

4. Vannucci A, Cavallone LF. Bedside predictors of difficult intubation: a systematic review. Minerva Anestesiol 2016;82:69-83.

5. Baxi V, Gaiwal S. Diffuse idiopathic skeletal hyperostosis of cervical spine - An unusual cause of difficult flexible fiber optic intubation. Saudi J Anaesth 2010;4:17-9.

6. Tack P, Victor J, Gemmel P, et al. 3D-printing techniques in a medical setting: a systematic literature review. Biomed Eng Online 2016;15:115.

7. Han B, Liu Y, Zhang X, et al. Three-dimensional printing as an aid to airway evaluation after tracheotomy in a patient with laryngeal carcinoma. BMC Anesthesiol 2016;16:6.

8. Varsak YK, Eryilmaz MA, Arbă̆ H. Dysphagia and airway obstruction due to large cervical osteophyte in a patient with ankylosing spondylitis. J Craniofac Surg 2014;25:1402-3.

9. Ozkalkanli MY, Katircioglu K, Ozkalkanli DT, et al. Airway management of a patient with Forestier's disease. J Anesth 2006;20:304-6.

10. Farooqi NA, Doran M, Buxton N. Cervical osteophytes: a cause of potentially life-threatening laryngeal spasms. Case report. J Neurosurg Spine 2006;4:419-20. 